$\exists \int$ Journal of Logic \& Analysis 2:7 (2010) 1-29

ISSN 1759-9008

\title{
Harmonic measure and subanalytically tame measures
}

\author{
TOBIAS KAISER
}

\begin{abstract}
We introduce the notion of analytically and subanalytically tame measures. These are measures which behave well in the globally subanalytic context; they preserve tameness: integrals of globally subanalytic functions with parameters resp. analytic functions with parameters restricted to globally subanalytic compact sets are definable in an o-minimal structure. We consider the harmonic measure for a semianalytic bounded domain in the plane. We show that the harmonic measure for such a domain is analytically tame if the angles at singular boundary points are irrational multiples of $\pi$. If the domain is a polygon and the angles at singular boundary points are rational or Diophantine irrational multiples of $\pi$ then the harmonic measure is subanalytically tame.
\end{abstract}

2000 Mathematics Subject Classification 03C64, 30C85, 32B20 (primary)

Keywords: semianalytic domain, harmonic measure, o-minimal structures

\section{Introduction}

Integration is a difficult task, both in model theory and in tame real geometry, since it is not a concept of first order. By integrating the simpliest functions, one immediately has to leave the semialgebraic or the globally subanalytic category, as the example $\log x=\int_{1}^{x} \frac{d t}{t}$ shows. We refer to Shiota [33] for semialgebraic and subanalytic sets and functions, and to Van den Dries and Miller [9] for the definition of globally subanalytic sets and functions. These are the sets resp. functions which are subanalytic in the ambient projective space. Bounded subanalytic sets are globally subanalytic. The logarithm on the positive real line is not globally subanalytic but definable in an o-minimal structure (see Wilkie [39], Van den Dries and Miller [8] and Van den Dries et. al. [7]; see also Van den Dries [6] for the general theory of o-minimal structures). O-minimal structures are defined via a finiteness property: the definable sets have only finitely many connected components. They are a generalization of the semialgebraic or globally subanalytic category sharing important tameness properties with them. Note that the subanalytic sets do not generate an o-minimal structure since, for example, the zero set of $\sin \pi t$ is $\mathbb{Z}$. But the globally subanalytic sets form an o-minimal structure 
denoted by $\mathbb{R}_{\mathrm{an}}$. O-minimal structures provide an excellent framework to capture important concepts from analysis and different classes of functions in the tame setting (see also Van den Dries and Speissegger [10, 11], Speissegger [37], Rolin et. al. [32], and [22]).

In this paper we investigate integration and measures in the globally subanalytic resp. o-minimal setting. So far the Lebesgue measure was considered in the literature. Taking the antiderivative of a one variable function preserves o-minimality (see [37]). In this generality the situation of integrating functions with parameters is unsettled. But there is the following very nice result of Comte, Lion and Rolin (see Comte et al. [4] and Lion and Rolin [26]): integrals of globally subanalytic functions with parameters with respect to the Lebesgue measure are definable in the o-minimal structure $\mathbb{R}_{\mathrm{an} \text {,exp }}$. The proof is based on the preparation theorem for globally subanalytic functions (see Lion and Rolin [25, 26]). $\mathbb{R}_{\mathrm{an}, \exp }$ is the structure generated by $\mathbb{R}_{\mathrm{an}}$ and the exponential function or, equivalently, the logarithm function; $\mathbb{R}_{\mathrm{an}, \exp }$ is again o-minimal by [8] and [7].

There are two possible ways to extend the results of Comte, Lion and Rolin one can think of. First, one can start with o-minimal expansions of $\mathbb{R}_{\mathrm{an}}$. But the only complete result in this direction is the one above. Soufflet $[35,36]$ has obtained partial results for the structure $\mathbb{R}_{\mathrm{an}}^{\mathbb{R}}$ which expands $\mathbb{R}_{\mathrm{an}}$ by all real power functions (see Miller [27] for more information on this structure). We pursue the second possible extension of the result of Comte, Lion and Rolin; we consider other measures. We make the following definition: a measure is called subanalytically tame if there is an o-minimal expansion of $\mathbb{R}_{\text {an }}$ such that integrals of globally subanalytic functions with parameters are definable in this o-minimal expansion. We also introduce the weaker notion of analytically tame measures: a measure is called analytically tame if there is an o-minimal expansion of $\mathbb{R}_{\mathrm{an}}$ such that integrals of analytic functions with parameters restricted to globally subanalytic compact sets are definable in this o-minimal expansion. See Definition 1.1 for the precise statement. By the result of Comte, Lion and Rolin the Lebesgue measure fits in this context. The question is now whether other significant measures fit.

An important class of measures is given by the harmonic measures. They are defined on the boundary of a bounded domain $\Omega$ in $\mathbb{R}^{n}$ and are intimately related to the Dirichlet problem for $\Omega$ (see Armitage and Gardiner [1, Chapter 6] for the definition of the harmonic measure and the Dirichlet problem, especially for the notion of generalized Dirichlet solution, the so called PWB solution; see also Garnett and Marshall [14] for harmonic measures on the plane): 
Let $z \in \Omega$ and let $\omega_{\Omega}(z,-)$ be the harmonic measure for $\Omega$ at $z$. Let $f$ be a continuous function on the boundary of $\Omega$. Then $\int_{\partial \Omega} f(\zeta) d \omega_{\Omega}(z, \zeta)=H_{f}(z)$, where $H_{f}(z)$ is the uniquely determined (generalized) Dirichlet solution of $f$; i.e. $H_{f}$ is harmonic in $\Omega$ and $H_{f}$ has a continuous extension to $\partial \Omega$ for all boundary points outside a small set of Lebesgue measure 0 and coincides there with $f$. We consider the plane case and assume that $\Omega$ is semianalytic (in dimension 2 subanalytic and semianalytic are the same, see Bierstone and Milman [2, Theorem 6.1]). In [21] it was shown, under the assumption that the angles of the domain at every singular boundary point is an irrational multiple of $\pi$, that the Dirichlet solution for a semianalytic boundary function is definable in the o-minimal structure $\mathbb{R}_{\mathcal{Q} \text {,exp }}$ (see [22] for the o-minimal structure $\mathbb{R}_{\mathcal{Q}}$ ). We use this result to show the following:

\section{Theorem A}

Let $\Omega$ be a bounded and semianalytic domain in the plane such that the angles at every singular boundary point are irrational multiple of $\pi$. Then for every $z \in \Omega$, the harmonic measure $\omega_{\Omega}(z,-)$ of $\Omega$ is analytically tame.

For certain polygons we can obtain subanalytic tameness. We call a polygon Diophantine if the angles at every singular boundary points are rational numbers or Diophantine irrational multiples of $\pi$. We prove

\section{Theorem B}

Let $P$ be a Diophantine polygon. Then for every $z \in P$, the harmonic measure $\omega_{P}(z,-)$ is subanalytically tame.

These two theorems are obtained as follows. We show first that the harmonic measure has density with respect to the length measure on the boundary similar to the case of a smooth boundary. For this we use the geometric tameness properties of globally subanalytic sets such as having stratification with nice features (see for example Kurdyka [23]). The density is related to the Green function $G_{z}$ given by $K_{z}-H_{K_{z}}$, where $K_{z}=-\log |z-w|$ is the Poisson kernel on $\mathbb{R}^{2}$. To obtain Theorem A we apply the results of $[21,22]$. To prove Theorem B we use the theory of Schwarz Christoffel maps (see Driscoll and Trefethen [12] and [18, Section 4]) and the subanalytic preparation theorem ([25, 26]; see also Parusinski [29, 30]). 


\section{Notation}

We denote by $\mathbb{N}$ the set of natural numbers and by $\mathbb{N}_{0}$ the set of nonnegative integers. Given $a \in \mathbb{C}$ and $r>0$, we denote by $B(a, r)$ the open disc and by $\bar{B}(a, r)$ the closed disc with center $a$ and radius $r$. We identify $\mathbb{R}^{2}$ with $\mathbb{C}$ in the usual way. A polydisc with center $a=\left(a_{1}, \ldots, a_{n}\right) \in \mathbb{C}^{n}$ is a set of the form $\prod_{i=1}^{n} B\left(a_{i}, r_{i}\right)$ where $r_{i}>0$. An open box with center $a=\left(a_{1}, \ldots, a_{n}\right) \in \mathbb{R}^{n}$ is a set of the form $\left.\prod_{i=1}^{n}\right] a_{i}-r_{i}, a_{i}+r_{i}[$ where $r_{i}>0$. Given a set $A \subset \mathbb{R}^{n}$ we denote by $\mathbb{1}_{A}$ the characteristic function of $A$. We call a nonempty, open and connected subset of $\mathbb{R}^{n}$ a domain.

\section{Definition of (sub)analytically tame measures}

We give the definition of a subanalytically tame measure and of an analytically tame measure. We show how the result of Comte, Lion and Rolin (see [4] and [26]) on the integration of globally subanalytic functions with parameters with respect to the Lebesgue measure fits precisely in this concept.

Definition 1.1 Let $n \in \mathbb{N}$ and let $\mu$ be a positive Borel measure on $\mathbb{R}^{n}$.

a) We call $\mu$ subanalytically tame if the following holds:

(i) For every globally subanalytic function $f: \mathbb{R}^{m} \times \mathbb{R}^{n} \rightarrow \mathbb{R}$ the set

$$
\infty(f, \mu):=\left\{x \in \mathbb{R}^{m}: \int_{\mathbb{R}^{n}}|f(x, t)| d \mu(t)=\infty\right\}
$$

is globally subanalytic.

(ii) There is an o-minimal expansion $\mathcal{R}$ of $\mathbb{R}_{\mathrm{an}}$ such that for every globally subanalytic function $f: \mathbb{R}^{m} \times \mathbb{R}^{n} \rightarrow \mathbb{R}$ the function

$$
\mathbb{R}^{m} \backslash \infty(f, \mu) \rightarrow \mathbb{R}, x \mapsto \int_{\mathbb{R}^{n}} f(x, t) d \mu(t),
$$

is definable in $\mathcal{R}$.

We call $\mathcal{R}$ a subanalytically integrating o-minimal structure of $\mu$. 
b) We call $\mu$ analytically tame if the following holds:

(i) For every real analytic function $f: U \rightarrow \mathbb{R}$ where $U \subset \mathbb{R}^{m} \times \mathbb{R}^{n}$ is open, and for every subanalytic set $K$ which is relatively compact in $U$ (i.e. $\bar{K}$ is compact and $\bar{K} \subset U$ ), the set

$$
\infty(f, K, \mu):=\left\{x \in \mathbb{R}^{m}: \int_{\mathbb{R}^{n}}\left|\mathbb{1}_{K} f(x, t)\right| d \mu(t)=\infty\right\}
$$

is globally subanalytic.

(ii) There is an o-minimal expansion $\mathcal{R}$ of $\mathbb{R}_{\text {an }}$ such that for every real analytic function $f: U \rightarrow \mathbb{R}$ where $U \subset \mathbb{R}^{m} \times \mathbb{R}^{n}$ is open, and for every subanalytic set $K$ which is relatively compact in $U$, the function

$$
\mathbb{R}^{m} \backslash \infty(f, K, \mu) \rightarrow \mathbb{R}, x \mapsto \int_{\mathbb{R}^{n}} \mathbb{1}_{K} f(x, t) d \mu(t),
$$

is definable in $\mathcal{R}$.

We call $\mathcal{R}$ an analytically integrating o-minimal structure of $\mu$.

Remark 1.2 A subanalytically tame measure $\mu$ is analytically tame and a subanalytically integrating o-minimal structure of $\mu$ is an analytically integrating o-minimal structure of $\mu$.

Remark 1.3 Let $\mu$ be a subanalytically (resp. analytically) tame measure. We denote by $\mathcal{R}_{\mu}$ the expansion of $\mathbb{R}_{\mathrm{an}}$ generated by all functions obtained in (ii) of Definition 1.1 a), and by $\mathcal{R}_{\mu, \text { an }}$ the expansion of $\mathbb{R}_{\mathrm{an}}$ generated by all functions obtained in (ii) of Definition $1.1 \mathrm{~b}$ ).

Note that $\mathcal{R}_{\mu, \text { an }}$ is a reduct of $\mathcal{R}_{\mu}$ if the latter one exists.

Examples 1.4 a) Let $a \in \mathbb{R}^{n}$. The Dirac measure $\delta_{a}$ is subanalytically tame and $\mathcal{R}_{\delta_{a}}=\mathcal{R}_{\delta_{a}, \text { an }}=\mathbb{R}_{\mathrm{an}}$

b) The Lebesgue measure $\lambda_{n}$ on $\mathbb{R}^{n}$ is subanalytically tame and $\mathcal{R}_{\lambda_{n}}=\mathbb{R}_{\mathrm{an} \text {,exp }}$ for all $n \in \mathbb{N}, \mathcal{R}_{\lambda_{n}, \text { an }}=\mathbb{R}_{\mathrm{an}, \text { exp }}$ if $n \geq 2$ and $\mathcal{R}_{\lambda_{1} \text {,an }}=\mathbb{R}_{\mathrm{an}}$. 
Proof a) This is obvious.

b) (i) $\mathcal{R}_{\lambda_{n}}=\mathbb{R}_{\mathrm{an}, \exp }$ for all $n \in \mathbb{N}$ :

By [4, Théorème 1'] (see also [26, Théorème 1]) the Lebesgue measure $\lambda_{n}$ on $\mathbb{R}^{n}$ is subanalytically tame with integrating o-minimal structure $\mathbb{R}_{\text {an,exp }}$. Let $f: \mathbb{R} \times \mathbb{R}^{n} \rightarrow \mathbb{R}$,

$$
f(x, t):= \begin{cases}\frac{1}{t_{1}} \quad \text { if } \quad 1 \leq t_{1} \leq x, \text { and } 0 \leq t_{i} \leq 1, i \neq 1, \\ 0 \quad \text { else. }\end{cases}
$$

Then $f$ is globally subanalytic, $\infty\left(f, \lambda_{n}\right)=\emptyset$, and

$$
\int_{\mathbb{R}^{n}} f(x, t)=\left\{\begin{array}{lll}
\log x & & x \geq 1, \\
& \text { if } & \\
0 & & x<1 .
\end{array}\right.
$$

So $\log :\left[1, \infty\left[\rightarrow \mathbb{R}\right.\right.$ is definable in $\mathcal{R}_{\lambda_{n}}$ and we get $\mathcal{R}_{\lambda_{n}}=\mathbb{R}_{\mathrm{an}, \mathrm{exp}}$.

(ii) $\mathcal{R}_{\lambda_{n} \text {,an }}=\mathbb{R}_{\text {an,exp }}$ for all $n \geq 2$ :

Let $K:=\left\{(x, t) \in \mathbb{R} \times \mathbb{R}^{n}: 0<x<1, x \leq t_{1} \leq 1,0<t_{2}<\frac{x}{t_{1}}, 0 \leq\right.$ $t_{i} \leq 1$ for $\left.3 \leq i \leq n\right\}$. Then $K$ is bounded and globally subanalytic. Let $f: \mathbb{R} \times \mathbb{R}^{n} \rightarrow \mathbb{R}, f \equiv 1$. Then for $0<x<1$

$$
\int \mathbb{1}_{K} f(x, t) \mathrm{dt}=\int_{t_{1}=x}^{1} \frac{x}{t_{1}} \mathrm{dt}_{1}=-x \log x .
$$

So $\log :] 0,1\left[\rightarrow \mathbb{R}\right.$ is definable in $\mathcal{R}_{\lambda_{n} \text {,an }}$ for $n \geq 2$ and we get the claim.

(iii) $\mathcal{R}_{\lambda_{1}, \text { an }}=\mathbb{R}_{\mathrm{an}}$ :

Let $f$ and $K$ be as in Definition 1.1. We can cover $\bar{K}$ by finitely many open boxes $Q_{1}, \ldots, Q_{k}$ such that for each $1 \leq i \leq k$ the real analytic function $f$ can be expanded into a power series around the center of $Q_{i}$ which converges on a neighbourhood of $\overline{Q_{i}}$. Since the intersection of open boxes is again an open box we may assume by the additivity of the Lebesgue measure that $U$ is an open box and that $f$ may be expanded into a power series which is convergent on a neighbourhood of $\bar{U}$. Since the Lebesgue measure is translation invariant we may assume that the center of $U$ is 0 . Let $f(x, t)=\sum_{k=0}^{\infty} a_{k}(x) t^{k}$ be the power series expansion of $f$ on 
$U$. Let $F(x, t):=\sum_{k=0}^{\infty} \frac{a_{k}(x)}{k+1} t^{k+1}$ be the antiderivative of $f$ with respect to $t$. Applying cell decomposition to $K$ (see [6, Chapter 3]) it is enough to deal with the case that there is a globally subanalytic set $A \subset \mathbb{R}^{m}$ and globally subanalytic functions $\varphi, \psi$ on $A$ with $\varphi<\psi$ such that

$$
K=(\varphi, \psi)_{A}:=\{(x, t) \in A \times \mathbb{R}: \varphi(x)<t<\psi(x)\} .
$$

Then $\int \mathbb{1}_{K} f(x, t) \mathrm{dt}=\int_{\varphi(x)}^{\psi(x)} f(x, t) \mathrm{dt}=F(x, \psi(x))-F(x, \varphi(x))$ and we get the claim.

Let $X \subset \mathbb{R}^{n}$ be a globally subanalytic set of dimension $k$. The canonical k-dimensional volume measure $\operatorname{vol}_{k, X}$ on $X$ is defined as follows. Choose a finite stratification $\left(X_{i}\right)_{i \in I}$ of $X$ and let $J:=\left\{i \in I \mid \operatorname{dim} \Gamma_{i}=k\right\}$. Then $\operatorname{vol}_{k, X}=\sum_{i \in J} \operatorname{vol}_{k, X_{i}}$. This does not depend on the chosen stratification.

Note that $\operatorname{vol}_{k, X}(X)<\infty$ if $X$ is bounded. This follows immediately by choosing a stratification such that the describing functions have bounded first derivatives (see [23, Remark 5.1]).

Proposition 1.5 Let $X$ be a globally subanalytic $C^{1}$-submanifold of $\mathbb{R}^{n}$ of dimension $k$. The canonical $k$-dimensional volume measure $\operatorname{vol}_{k, X}$ on $X$ is subanalytically tame with

$$
\begin{gathered}
\mathcal{R}_{\mathrm{vol}_{k, X}}=\left\{\begin{array}{lll}
\mathbb{R}_{\mathrm{an}, \text { exp }} & & k \geq 1, \\
& \text { if } & k=0, \\
\mathbb{R}_{\mathrm{an}} & & k=0
\end{array}\right. \\
\mathcal{R}_{\mathrm{vol}_{k, X}, \text { an }}=\left\{\begin{array}{lll}
\mathbb{R}_{\mathrm{an}, \exp } & & k \geq 2, \\
& \text { if } & \\
\mathbb{R}_{\mathrm{an}} & & k=0,1 .
\end{array}\right.
\end{gathered}
$$

Proof If $k=n$ then $\operatorname{vol}_{k, X}$ equals the Lebesgue measure restricted to the open globally subanalytic set $X$ and we get the claim by Example 1.4 b). If $k=0$ we get the claim by Example 1.4 a). So we assume that $0<k<n$. By stratification (see for example [23, Theorem A and Remark 5.1]) there are globally subanalytic subsets $X_{1}, \ldots, X_{\ell}$ of $X$ with $\operatorname{dim}\left(X \backslash \bigcup_{1 \leq i \leq \ell} X_{i}\right)<k$ such that for each $i \in\{1, \ldots, \ell\}$ the following holds. 
There is an orthogonal map $A_{i}$ such that $A_{i}\left(X_{i}\right)=\operatorname{graph}\left(f_{i}\right)$, where $f_{i}: U_{i} \rightarrow \mathbb{R}^{n-k}$ is a globally subanalytic and real analytic map, and where $U_{i} \subset \mathbb{R}^{k}$ is a globally subanalytic domain. Let $g_{i}: U_{i} \rightarrow \mathbb{R}^{n}, g_{i}(x):=\left(x, f_{i}(x)\right)$. Then the canonical $k$-dimensional volume measure $\operatorname{vol}_{k, \Gamma_{i}}$ on $\Gamma_{i}:=\operatorname{graph}\left(f_{i}\right)$ is given by $\sqrt{\operatorname{det}\left({ }^{t} D g_{i}(x) D g_{i}(x)\right)} \lambda_{k}(x)$, where $D g_{i}(x)$ denotes the Jacobian of $g_{i}$ in $x \in U_{i}$. Since $\sqrt{\operatorname{det}\left({ }^{t} D g_{i}(x) D g_{i}(x)\right)}$ is globally subanalytic, strictly positive and real analytic on $U_{i}$ we get by the proof of Example 1.4 b) that $\mathcal{R}_{\mathrm{vol}_{k, \Gamma_{i}}}=\mathbb{R}_{\mathrm{an}, \text { exp }}$ if $k>0$, that $\mathcal{R}_{\mathrm{vol}_{k, \Gamma_{i}} \text {, an }}=\mathbb{R}_{\mathrm{an}, \exp }$ if $k \geq 2$ and that $\mathcal{R}_{\mathrm{vol}_{k, \Gamma_{i}} \text {,an }}=\mathbb{R}_{\mathrm{an}}$ if $k=1$. Since

$$
\operatorname{vol}_{k, X}=\sum_{i=1}^{\ell} \operatorname{vol}_{k, X_{i}}=\sum_{i=1}^{\ell} \operatorname{vol}_{k, \Gamma_{i}} \circ A_{i}^{-1}
$$

we obtain the claim.

\section{The harmonic measure of a bounded semianalytic domain in the plane}

Let $\Omega$ be a semianalytic and bounded domain in the plane and, for $z \in \Omega$, let $\omega_{\Omega}(z,-)$ be its harmonic measure with respect to $z$. We want to analyze these measures with regard to analytical and subanalytic tameness. Since $\omega_{\Omega}(z,\{x\})=0$ for every point $x \in \partial \Omega$ (see [1, Theorem 6.5.5 and Example 5.1.2]), the harmonic measure does not change if we remove the finitely many isolated boundary points. Hence we may assume that $\Omega$ has no isolated boundary points. This reliefs the technical work at some points. For example, by [19, Theorem 2.4] every continuous boundary function has a classical Dirichlet solution; i.e. given $h \in C(\partial \Omega)$ there is a unique $u \in \mathcal{H}(\Omega) \cap C(\bar{\Omega})$ such that $u=h$ on $\partial \Omega$. Here $\mathcal{H}(\Omega)$ denotes the space of harmonic functions in $\Omega$.

From now on $\Omega$ denotes a semianalytic and bounded domain in $\mathbb{R}^{2}$ without isolated boundary points. Let $z \in \Omega$. We want to show that the harmonic measure $\omega_{\Omega}(z,-)$ fulfils condition (i) of Definition $1.1 \mathrm{a}$ ), and we want to give a description of the harmonic measure similar to the case of domains with analytically smooth boundary (see [14, Theorem II.2.5]). Therefore, we use the results of [20] and [22]. Let $\operatorname{Sing}(\partial \Omega$ ) be the set of singular boundary points of $\Omega$. We assume that $\varangle(\Omega, x) \subset \mathbb{R} \backslash\{0\}$ for all $x \in \operatorname{Sing}(\partial \Omega)$. Here $\varangle(\Omega, x)$ denotes the set of angles of the finitely many connected components of the germ of $\Omega$ at $x$ which have $x$ as a singular boundary point (compare with [21, Definition 2.3]). 
We start with the analytically smooth case to see the kind of arguments we will use.

Remark 2.1 (see [14, Corollary II.2.6]) Assume that $\partial \Omega$ is analytically smooth. Let $z \in \Omega$. Then

$$
\omega_{\Omega}(z,-)=\frac{1}{2 \pi} \frac{\partial G_{z}}{\partial n} d \sigma
$$

Here $\sigma:=\operatorname{vol}_{1, \partial \Omega}$ is the length measure of $\partial \Omega, G_{z}$ is the Green function with pole $z$, and $n$ denotes the inner normal on $\partial \Omega$ with respect to $\Omega$.

Using Proposition 1.5, we obtain immediately that the harmonic measure is subanalytically tame if the domain has analytically smooth boundary:

Corollary 2.2 Assume that $\partial \Omega$ is analytically smooth. Then the harmonic measure $\omega_{\Omega}(z,-)$ is subanalytically tame with $\mathcal{R}_{\omega_{\Omega}(z,-)}=\mathbb{R}_{\mathrm{an}, \exp }$ and $\mathcal{R}_{\omega_{\Omega}(z,-), \text { an }}=\mathbb{R}_{\mathrm{an}}$.

Proof Let $\sigma$ be the length measure on $\partial \Omega$. By Remark 2.1, the restriction of $\omega_{\Omega}(z,-)$ to $\partial \Omega$ has density with respect to $\sigma$. The density function is given by $\frac{1}{2 \pi} \frac{\partial G_{z}}{\partial n}$. It is real analytic and strictly positive on $\partial \Omega$ by [14, Corollary II.2.6]). Therefore we get the corollary from Proposition 1.5.

Next, we leave aside the assumption that $\partial \Omega$ is analytically smooth and show that property (i) of Definition 1.1 a) holds for the harmonic measure $\omega_{\Omega}(z,-), z \in \Omega$.

Proposition 2.3 Assume that $\varangle(\Omega, x) \subset \mathbb{R} \backslash\{0\}$ for all $x \in \operatorname{Sing}(\partial \Omega)$. Let $z \in \Omega$ and let $f: \mathbb{R}^{m} \times \partial \Omega \rightarrow \mathbb{R}_{\geq 0}$ be globally subanalytic. Then

$$
\infty\left(f, \omega_{\Omega}(z,-)\right)=\left\{x \in \mathbb{R}^{m}: \int_{\partial \Omega} f(x, \xi) d \omega_{\Omega}(z, \xi)=\infty\right\}
$$

is globally subanalytic.

Proof We do the proof in the case that $\Omega$ is simply connected. With this restriction the technical arguments of the proof can be followed more easily. We use here that by the Riemann Mapping Theorem there is a biholomorphic map from the upper half plane $\mathbb{H}$ to $\Omega$. In the general case, the domain is mapped biholomorphically on a domain with analytically smooth boundary, and the same ideas work.

By replacing $f$ by $f+1$ (note that $\int_{\partial \Omega} 1 d \omega_{\Omega}(z, \xi)=1$, see for example [1, Theorem 6.4.1]), we may assume that $f>0$. We choose a finite stratification $\mathcal{T}$ of $\partial \Omega$ (see 
[23]) such that $\bar{T} \cap \operatorname{Sing}(\partial \Omega)$ contains at most one point for all $T \in \mathcal{T}$. Let $T \in \mathcal{T}$ with $\operatorname{dim} T=1$. It is enough to show that

$$
\left\{x \in \mathbb{R}^{m}: \int_{T} f(x, \xi) d \omega_{\Omega}(z, \xi)=\infty\right\}
$$

is globally subanalytic. Let $\{a, b\}:=\bar{T} \backslash T$; we may assume that at most $a \in \operatorname{Sing}(\partial \Omega)$. Moreover, we may assume that $a=0$.

Since $\Omega$ is simply connected there is, by Caratheodory's Prime End Theorem (see Pommerenke [31, Chapter 2, p. 18]) and by the curve selection lemma (see for example [6, p. 94]), a biholomorphic map $F: \mathbb{H} \rightarrow \Omega$ (where $\mathbb{H}$ denotes the upper half plane) such that $F$ has a continuous extension to $\partial \mathbb{H}$ with $F(0)=0$ and such that $F:] 0, \gamma[\stackrel{\cong}{\rightrightarrows} T$ is homeomorphic for some $\gamma \in \mathbb{R}$. We deal with the case $\gamma>0$, the other case is treated similarly. By conformal invariance of the harmonic measure (see Garnett $\left[14\right.$, p. 2]) we have $\omega_{\Omega}(z,-)=\omega_{\mathbb{H}}\left(z^{*},-\right) \circ F^{-1}$ where $z^{*}:=F^{-1}(z)$. We obtain therefore that

$$
\int_{T} f(x, \xi) d \omega_{\Omega}(z, \xi)=\int_{0}^{\gamma} f(x, F(t)) d \omega_{\mathbb{H}}\left(z^{*}, t\right) d t=\int_{0}^{\gamma} f(x, F(t)) h_{z^{*}}(t) d t
$$

where $h_{z^{*}}(t)=\frac{\operatorname{Im} z^{*}}{\left|z^{*}-t\right|^{2}}($ see $[1,1.7])$. Note that $h_{z^{*}}$ is real analytic on $\mathbb{R}$. We set

$$
A:=\left\{(x, \xi) \in \mathbb{R}^{m} \times \bar{T}: \varlimsup_{\substack{\eta \rightarrow \xi \\ \eta \in T}} f(x, \eta)=\infty\right\}
$$

Then $A$ is globally subanalytic and, by the uniform finiteness property in o-minimal structures (see [6, p. 53]), there is a natural number $N$ such that $\{\xi \in \bar{T} \mid(x, \xi) \in A\}$ has at most $N$ elements for every $x \in \mathbb{R}^{m}$. By cell decomposition (see [6, Chapter 3]), there is a partition $\mathbb{R}^{m}=\bigcup_{1 \leq j \leq k} X_{j}$ of $\mathbb{R}^{m}$ into globally subanalytic sets $X_{j}$ such that for $j \in\{1, \ldots, k\}$, there are globally subanalytic and continuous functions $\alpha_{j \ell}: X_{j} \rightarrow \bar{T}, 1 \leq \ell \leq r_{j}$, such that

$$
\left\{(x, \xi) \in X_{j} \times \bar{T}: \varlimsup_{\substack{\eta \rightarrow \xi \\ \eta \in T}} f(x, \eta)=\infty\right\}=\bigcup_{1 \leq \ell \leq r_{j}} \operatorname{graph}\left(\alpha_{j \ell}\right) .
$$

We fix $j \in\{1, \ldots, k\}$ and omit this subscript. We choose an orientation on $\bar{T}$; this induces an ordering $<$ on $T \cong] 0, \gamma[$. We may choose the orientation such that

$$
0=a \leq \alpha_{1}(x)<\alpha_{2}(x)<\ldots<\alpha_{r}(x) \leq b
$$


for all $x \in X$. After choosing a suitable subpartition of $\mathbb{R}^{m}$, we may assume that either $\alpha_{1}(x)=a$ or $\alpha_{1}(x)>a$ for all $x \in X$ and either $\alpha_{r}(x)=b$ or $\alpha_{r}(x)<b$ for all $x \in X$. We distinguish four cases:

Case 1: $\alpha_{1}=a$ and $\alpha_{r}=b$.

Case 2: $\alpha_{1}=a$ and $\alpha_{r}<b$.

Case 3: $\alpha_{1}>a$ and $\alpha_{r}=b$.

Case 4: $\alpha_{1}>a$ and $\alpha_{r}<b$.

We deal with Case 2; the other cases can be handled in a similar way. We choose globally subanalytic and continuous functions $\beta_{\ell}: X \rightarrow T, 1 \leq \ell \leq r$, such that

$$
a=\alpha_{1}(x)<\beta_{1}(x)<\alpha_{2}(x)<\ldots<\alpha_{r}(x)<\beta_{r}(x)<b
$$

for all $x \in X$. Then

$$
\begin{aligned}
& \left\{x \in X: \iint_{T} f(x, \xi) d \omega_{\Omega}(z, \xi)=\infty\right\} \\
& =\bigcup_{\ell=1}^{r}\left\{x \in X: \int_{\left[\alpha_{\ell}(x), \beta_{\ell}(x)\right]} f(x, \xi) d \omega_{\Omega}(z, \xi)=\infty\right\} \\
& \cup \bigcup_{\ell=1}^{r-1}\left\{x \in X: \int_{\left[\beta_{\ell}(x), \alpha_{\ell+1}(x)\right]} f(x, \xi) d \omega_{\Omega}(z, \xi)=\infty\right\}
\end{aligned}
$$

(where $\left[\alpha_{\ell}(x), \beta_{\ell}(x)\right]:=\left\{\xi \in \bar{T}: \alpha_{\ell}(x) \leq \xi \leq \beta_{\ell}(x)\right\}$ and $\left[\beta_{\ell}(x), \alpha_{\ell+1}(x)\right]:=\left\{\xi \in \bar{T}: \beta_{\ell}(x)\right.$ $\left.\left.\leq \xi \leq \alpha_{\ell+1}(x)\right\}\right)$.

We fix $1 \leq \ell \leq r$ and we investigate the integral

$$
\int_{\left[\alpha_{\ell}(x), \beta_{\ell}(x)\right]} f(x, \xi) d \omega_{\Omega}(z, \xi) .
$$

By the subanalytic preparation theorem (see [25, Théorème 1] and [26, Section 3]) or by Miller [27, Proposition 5.2], we can partition $X$ into finitely many globally subanalytic sets $Y_{p}, 1 \leq p \leq q$, such that for each $p$ there is $\nu_{p} \in \mathbb{Q}_{<0}$ with $f(x, \xi) \sim\left|\xi-\alpha_{\ell}(x)\right|^{\nu_{p}}$ 
as $\xi \searrow \alpha_{\ell}(x)$; i.e. $\lim _{\xi \backslash \alpha_{\ell}(x)} \frac{f(x, \xi)}{\left|\xi-\alpha_{\ell}(x)\right|^{\nu_{p}}} \neq 0$ for all $x \in Y_{p}$. Let $x \in Y_{p}$. We have by $(*)$

$$
\int_{\left[\alpha_{\ell}(x), \beta_{\ell}(x)\right]} f(x, \xi) d \omega_{\Omega}(z, \xi)=\int_{\gamma_{\ell}(x)}^{\delta_{\ell}(x)} f(x, F(t)) h_{z^{*}}(t) d t
$$

where $\gamma_{\ell}(x):=F^{-1}\left(\alpha_{\ell}(x)\right)$ and $\delta_{\ell}(x):=F^{-1}\left(\beta_{\ell}(x)\right)$.

Let $\ell>1$. Since $\partial \Omega$ is analytic in $\alpha_{\ell}(x)$ and since $\partial \mathbb{H}$ is analytic we obtain, by the Schwarz reflection principle (see for example [20, Example 2.13]), that $F$ has a holomorphic extension to a neighbourhood of $\gamma_{\ell}(x)$ with $F^{\prime}\left(\gamma_{\ell}(x)\right) \neq 0$. Hence we get that $\left|F(t)-F\left(\gamma_{\ell}(x)\right)\right| \sim\left|t-\gamma_{\ell}(x)\right|$ as $t \rightarrow \gamma_{\ell}(x)$. Therefore

$$
\left\{x \in Y_{p}: \int_{\left[\alpha_{\ell}(x), \beta_{\ell}(x)\right]} f(x, \xi) d \omega_{\Omega}(z, \xi)=\infty\right\}=\left\{\begin{array}{ccc}
Y_{p} & \nu_{p} \leq-1, \\
& \text { if } & \\
\emptyset & & \nu_{p}>-1 .
\end{array}\right.
$$

Let $\ell=1$. By [20, Theorem 2.18 and Remark 2.19] (see also Lehman [24, Theorem 1]), we find some $\mu>0$ such that $|F(t)| \sim t^{\mu}$ as $t \rightarrow 0$. Therefore

$$
\left\{x \in Y_{p}: \int_{\left[0, \beta_{0}(x)\right]} f(x, \xi) d \omega_{\Omega}(z, \xi)=\infty\right\}=\left\{\begin{array}{ccc}
Y_{p} & \nu_{p} \mu \leq-1, \\
& \text { if } & \\
\emptyset & & \nu_{p} \mu>-1 .
\end{array}\right.
$$

Since $Y_{p}$ was arbitrary we get that

$$
\left\{x \in X: \int_{\left[\alpha_{\ell}(x), \beta_{\ell}(x)\right]} f(x, \xi) d \omega_{\Omega}(z, \xi)=\infty\right\}
$$

is globally subanalytic for every $\ell$. With the same argument we can show this for $\left[\beta_{\ell}(x), \alpha_{\ell+1}(x)\right]$ and obtain that

$$
\left\{x \in X: \int_{T} f(x, \xi) d \omega_{\Omega}(z, \xi)=\infty\right\}
$$

is globally subanalytic. Therefore Case 2 is settled. The other cases are treated similarly and we get the claim. 
If $\Omega$ has analytically smooth boundary we know by Remark 2.1 , that the harmonic measure $\omega_{\Omega}(z,-)$ is given by $\frac{1}{2 \pi} \frac{\partial G}{\partial n} \sigma$ where $G:=G_{z}$ is the Green function with pole $z, \sigma$ is the length measure of $\partial \Omega$, and $n$ is the inner normal relative to $\Omega$. We want to generalize this formula to semianalytic domains with singularities. We assume again that $\Omega \subset \mathbb{R}^{2}$ is a semianalytic and bounded domain without isolated boundary points such that $\varangle(\Omega, x) \subset \mathbb{R} \backslash\{0\}$ for all singular boundary points $x \in \operatorname{Sing}(\partial \Omega)$. We need some technical preparation to formulate a rigorous statement and to give a rigorous proof of it.

Let $x \in \partial \Omega \backslash \operatorname{Sing}(\partial \Omega)$. Then the germ of $\Omega$ at $x$ has either one or two components.

Definition 2.4 We set

$$
\begin{aligned}
& \partial_{1} \Omega:=\{x \in \partial \Omega \backslash \operatorname{Sing}(\partial \Omega): \text { the germ of } \Omega \text { at } x \text { has one component }\}, \\
& \partial_{2} \Omega:=\{x \in \partial \Omega \backslash \operatorname{Sing}(\partial \Omega): \text { the germ of } \Omega \text { at } x \text { has two components }\} .
\end{aligned}
$$

Examples 2.5 a) Let $\Omega:=B(0,1) \backslash \mathbb{R}_{\leq 0}$. Then $\operatorname{Sing}(\partial \Omega)=\{0,-1\}, \partial_{1} \Omega=$ $\partial B(0,1) \backslash\{-1\}$ and $\left.\partial_{2}(\Omega)=\right]-1,0[$.

b) Let $\Omega:=B(0,1) \backslash\left[-\frac{1}{2}, \frac{1}{2}\right]$. Then $\operatorname{Sing}(\partial \Omega)=\left\{-\frac{1}{2}, \frac{1}{2}\right\}, \partial_{1} \Omega=\partial B(0,1)$ and $\left.\partial_{2} \Omega=\right]-\frac{1}{2}, \frac{1}{2}[$.

We have $\partial \Omega \backslash \operatorname{Sing}(\partial \Omega)=\partial_{1} \Omega \dot{\cup} \partial_{2} \Omega$. Let $\Gamma$ be a component of $\partial \Omega \backslash \operatorname{Sing}(\partial \Omega)$. Then $\Gamma \subset \partial_{1} \Omega$ or $\Gamma \subset \partial_{2} \Omega$. If $\Gamma \subset \partial_{1} \Omega$ then the inner normal $n$ on $\Gamma$ with respect to $\Omega$ is well defined. If $\Gamma \subset \partial_{2} \Omega$ we find open sets $U_{+}, U_{-} \subset \Omega$ with $U_{+} \cap U_{-}=\emptyset$ and $\Gamma \subset \overline{U_{+}} \cap \overline{U_{-}}$. We denote the inner normal on $\Gamma$ with respect to $U_{+}$by $n_{+}$and the inner normal on $\Gamma$ with respect to $U_{-}$by $n_{-}$. Let $h$ be a continuous function on $\partial \Omega$ that is real analytic on $\partial \Omega \backslash \operatorname{Sing}(\partial \Omega)$. Let $u$ be the Dirichlet solution for $h$. If $\Gamma \subset \partial_{1} \Omega$ then $u$ has a harmonic extension to a neighbourhood of $\Gamma$ by reflection. Therefore $\frac{\partial u}{\partial n}$ exists. If $\Gamma \subset \partial_{2} \Omega$ then $\left.u\right|_{U_{+}}$has a harmonic extension to a neighbourhood of $\Gamma$ again by reflection. Therefore $\frac{\partial u}{\partial n_{+}}$exists and is well defined. The same holds for $\frac{\partial u}{\partial n_{-}}$. Since $G_{z}=K_{z}-H_{K_{z}}$ and $K_{z}$ is harmonic on $\mathbb{R}^{2} \backslash\{z\}$ the same holds for $G_{z}$.

Finally, we set $\sigma_{1}:=\left.\sigma\right|_{\partial \Omega_{1}}$ and $\sigma_{2}:=\left.\sigma\right|_{\partial \Omega_{2}}$, where $\sigma=\operatorname{vol}_{1, \partial \Omega}$ is the length measure on $\partial \Omega$. 
Tobias Kaiser

We obtain

Proposition 2.6 Assume that $\varangle(\Omega, x) \subset \mathbb{R} \backslash\{0\}$ for all $x \in \operatorname{Sing}(\partial \Omega)$. Let $z \in \Omega$. Then

$$
\omega_{\Omega}(z,-)=\frac{1}{2 \pi}\left(\frac{\partial G_{z}}{\partial n} \sigma_{1}+\left(\frac{\partial G_{z}}{\partial n_{+}}+\frac{\partial G_{z}}{\partial n_{-}}\right) \sigma_{2}\right) .
$$

Proof We choose a stratification $\mathcal{T}$ of $\partial \Omega$ (see [23]) and, for all sufficiently small $\varepsilon>0$, we choose semianalytic domains $\Omega_{\varepsilon}$ with $C^{2}$ boundary such that $\overline{\Omega_{\varepsilon}} \subset \Omega$ and the following properties are fulfilled:

(i) For every $T \in \mathcal{T}$ with $\operatorname{dim} T=1$ there is $a_{T} \in \mathbb{R}^{2}$ with $\left|a_{T}\right|=1$ such that the following holds.

Let $V_{T, \varepsilon}:=\bigcup_{x \in \bar{T} \backslash T} \bar{B}(x, \varepsilon)$.

If $T \subset \partial_{1} \Omega$ then there is $\left.\lambda_{T, \varepsilon} \in\right] 0, \varepsilon$ [ such that $T_{\varepsilon}:=T \backslash V_{T, \varepsilon}+\lambda_{T, \varepsilon} a_{T} \subset \partial \Omega_{\varepsilon}$.

If $T \subset \partial_{2} \Omega$ then there are $\left.\lambda_{T,+, \varepsilon} \in\right] 0, \varepsilon\left[\right.$ and $\left.\lambda_{T,-, \varepsilon} \in\right]-\varepsilon, 0[$ such that $T_{\varepsilon,+}:=T \backslash V_{T, \varepsilon}+\lambda_{T,+, \varepsilon} a_{T} \subset \partial \Omega_{\varepsilon}$ and $T_{\varepsilon,-}:=T \backslash V_{T, \varepsilon}+\lambda_{T,-, \varepsilon} a_{T} \subset \partial \Omega_{\varepsilon}$.

(ii) Let $\partial_{*} \Omega_{\varepsilon}:=\partial \Omega_{\varepsilon} \backslash\left(\bigcup_{T \subset \partial_{1} \Omega} T_{\varepsilon} \cup \bigcup_{T \subset \partial_{2} \Omega}\left(T_{\varepsilon,+} \cup T_{\varepsilon,-}\right)\right)$. Then $\partial_{*} \Omega_{\varepsilon} \subset$ $\bigcup_{\{x\} \in \mathcal{T}} B(x, \varepsilon)$ and $\operatorname{vol}_{1}\left(\partial_{*} \Omega_{\varepsilon}\right)<\varepsilon$.

For all sufficiently small $\varepsilon>0$ we have $\bar{B}(z, \varepsilon) \subset \Omega_{\varepsilon}$. Let $U_{\varepsilon}:=\Omega_{\varepsilon} \backslash \bar{B}(z, \varepsilon)$. Let $h$ be a real polynomial on $\mathbb{R}^{2}$ and let $u$ be the Dirichlet solution for $\left.h\right|_{\partial \Omega}$. Since $U_{\varepsilon}$ has $C^{2}$ boundary and since $u$ and $G$ are $C^{2}$ on a neighbourhood of $\overline{U_{\varepsilon}}$ we can apply Green's formula (see for example Helms [15, p. 7]) to obtain

$$
\int_{U_{\varepsilon}}(u \Delta G-G \Delta u) d x=\int_{\partial U_{\varepsilon}}\left(u \frac{\partial G}{\partial n_{\varepsilon}}-G \frac{\partial u}{\partial n_{\varepsilon}}\right) d \tau_{\varepsilon},
$$

where $\tau_{\varepsilon}$ denotes the length measure on $\partial U_{\varepsilon}$ and $n_{\varepsilon}$ denotes the inner normal on $\partial U_{\varepsilon}$ with respect to $U_{\varepsilon}$. The integral on the left equals 0 since $G$ and $u$ are harmonic on $U_{\varepsilon}$, and we get

$$
0=\int_{\partial \Omega_{\varepsilon}} u \frac{\partial G}{\partial n_{\varepsilon}} d \sigma_{\varepsilon}-\int_{\partial B(z, \varepsilon)} u \frac{\partial G}{\partial \widetilde{n}_{\varepsilon}} d \sigma_{\varepsilon}^{\prime}-\int_{\partial \Omega_{\varepsilon}} G \frac{\partial u}{\partial n_{\varepsilon}} d \sigma_{\varepsilon}+\int_{\partial B(z, \varepsilon)} G \frac{\partial u}{\partial \widetilde{n}_{\varepsilon}} d \sigma_{\varepsilon}^{\prime}
$$

where $\sigma_{\varepsilon}$ denotes the length measure on $\partial \Omega_{\varepsilon}, \sigma_{\varepsilon}^{\prime}$ the length measure on $\partial B(z, \varepsilon)$ and $\widetilde{n}_{\varepsilon}$ the inner normal on $\partial B(z, \varepsilon)$ relative to $B(z, \varepsilon)$. By classical arguments (see for example [15, pp. 9-11]) the second integral converges to $2 \pi u(z)$ and the fourth integral to 0 as $\varepsilon \rightarrow 0$. 
We consider the first integral as $\varepsilon \rightarrow 0$. The behaviour of the integrand (especially of $\frac{\partial G}{\partial n_{\varepsilon}}$ ) at the zero dimensional strata is critical. Let $x \in \partial \Omega$ with $\{x\} \in \mathcal{T}$. Without restriction we may assume that $x=0$.

Case 1: $0 \notin \operatorname{Sing}(\partial \Omega)$. We know that $G$ can be extended analytically to a neighbourhood of 0 and therefore $\nabla G$ is bounded on a neighbourhood of 0 .

Case 2: $0 \in \operatorname{Sing}(\partial \Omega)$. Using [21, Theorem 2.4 and Theorem 3.2] (see also Wasow [38, Theorem 5]) we find $\alpha>-1$ such that $|\nabla G(y)|$ is bounded by $|y|^{\alpha}$ on $\Omega$ near 0 .

By the estimates for $\nabla G$ obtained in Cases 1 and 2 and the fact that $G$ can be analytically extended across $\partial \Omega \backslash \operatorname{Sing}(\partial \Omega)$ and that $u$ can be continuously extended to $\partial \Omega$ by $h$, we can apply Lebesgue's dominated convergence theorem and get for the first integral by properties (i) and (ii) of $\partial \Omega_{\varepsilon}$ that

$$
\lim _{\varepsilon \rightarrow 0} \int_{\partial \Omega_{\varepsilon}} u \frac{\partial G}{\partial n_{\varepsilon}} d \sigma_{\varepsilon}=\int_{\partial \Omega_{1}} h \frac{\partial G}{\partial n} d \sigma_{1}+\int_{\partial \Omega_{2}} h\left(\frac{\partial G}{\partial n_{+}}+\frac{\partial G}{\partial n_{-}}\right) d \sigma_{2} .
$$

Finally, we consider the third integral. With the same arguments as above, we obtain similar estimates for $\nabla u$. Using the fact that $u$ can be extended analytically across $\partial \Omega \backslash \operatorname{Sing}(\partial \Omega)$ and that $\left.G\right|_{\partial \Omega}=0$, we get

$$
\lim _{\varepsilon \rightarrow 0} \int_{\partial \Omega_{\varepsilon}} G \frac{\partial u}{\partial n} d \sigma_{\varepsilon}=0
$$

Hence

$$
\int_{\partial \Omega_{1}} h \frac{\partial G}{\partial n} d \sigma_{1}+\int_{\partial \Omega_{2}} h\left(\frac{\partial G}{\partial n_{+}}+\frac{\partial G}{\partial n_{-}}\right) d \sigma_{2}=2 \pi u(z) .
$$

Since the real polynomials are dense in $C(\partial \Omega)$, the proposition follows. 


\section{3 (Sub)analytical tameness of the harmonic measure}

Next, we want to prove Theorem A. We use the following results. In [21] it was shown that given a bounded and semianalytic domain in the plane without isolated boundary points and given a continuous and semianalytic function on the boundary, the Dirichlet solution is definable in the o-minimal structure $\mathbb{R}_{\mathcal{Q} \text {,exp }}$ if $\varangle(\Omega, x) \subset(\mathbb{R} \backslash \mathbb{Q}) \pi$ for all singular boundary points $x \in \partial \Omega$. The o-minimal structure $\mathbb{R}_{\mathcal{Q}}$ was introduced in [22] and is related to Hilbert's $16^{\text {th }}$ problem. It is generated by a certain quasianalytic class which was introduced by Ilyashenko (see Ilyashenko [17]) in his work on Hilbert's $16^{\text {th }}$ problem. Functions from this quasianalytic class $\mathcal{Q}$ are holomorphic on certain subsets of the Riemann surface of the logarithm, so-called quadratic domains. We use the results and notations of [22] and [21], especially the properties of the quasianalytic classes that generate the o-minimal structure $\mathbb{R}_{\mathcal{Q}}$, to show our first main theorem:

Theorem A Let $\Omega \subset \mathbb{R}^{2}$ be a bounded and semianalytic domain. Assume that $\varangle(\Omega, x) \subset(\mathbb{R} \backslash \mathbb{Q}) \pi$ for all $x \in \operatorname{Sing}(\partial \Omega)$. Let $z \in \Omega$. Then the harmonic measure $\omega_{\Omega}(z,-)$ is analytically tame with analytically integrating o-minimal structure $\mathbb{R}_{\mathcal{Q}}$.

Proof We may assume that $\Omega$ has no isolated boundary points (compare with the discussion at the beginning of section 2). By stratification (see [23]) and by the fact that globally subanalytic functions in one variable are locally given by puiseux series (see [5, p. 192])), we find a globally subanalytic stratification $\mathcal{T}$ of $\partial \Omega$ of the following kind.

Let $T \in \mathcal{T}$ with $\operatorname{dim} T=1$. Then the following holds:

(i) There are real power series $\psi, \chi$ convergent on $]-1-\varepsilon, 1+\varepsilon$ [ for some $\varepsilon>0$ such that $T=A \circ \varphi(] 0,1[)$ where $\varphi:=(\psi, \chi)$ and where $A$ is an orthogonal coordinate transformation. Moreover, $\varphi(t) \in \partial \Omega$ and $\varphi^{\prime}(t) \neq(0,0)$ for all $t \in] 0,1+\varepsilon[$.

(ii) At most $\varphi(0) \in \operatorname{Sing}(\partial \Omega)$.

It is enough to show for every $T \in \mathcal{T}$ with $\operatorname{dim} T=1$ that $\mu_{T}:=\left.\omega_{\Omega}(z,-)\right|_{T}$ is analytically tame with analytically integrating o-minimal structure $\mathbb{R}_{\mathcal{Q}}$. So let $T \in \mathcal{T}$ with $\operatorname{dim} T=1$. We may assume that $\varphi(0)=0$. We may also assume for convenience that $T \subset \partial_{1} \Omega$ (the general case is treated similarly). Then $\mu_{T}=\left.\frac{1}{2 \pi} \frac{\partial G}{\partial n} \sigma\right|_{T}$ where 
$G=G_{z}$ and $\sigma$ is the length measure of $\partial \Omega$ (see Proposition 2.6). Let $f: U \rightarrow \mathbb{R}$ be real analytic, where $U \subset \mathbb{R}^{m} \times \mathbb{R}^{2}$ is open, and let $K$ be a globally subanalytic and relatively compact subset of $U$. Using the estimates for $|\nabla G|$ in the proof of Proposition 2.6, we see that $\infty\left(f, K, \mu_{T}\right)=\emptyset$. We work in the coordinate system induced by $A$; i.e., we assume by applying some rotation if necessary that $A=\mathrm{Id}$. We may also assume that $T \subset \mathbb{C} \backslash \mathbb{R}_{\leq 0}$. We set $\varphi_{\perp}^{\prime}(t):=\left(-\chi^{\prime}(t), \psi^{\prime}(t)\right)$. Then the inner normal at $\varphi(t) \in T$ is given by $\rho \frac{\varphi_{\perp}^{\prime}(t)}{\left|\varphi_{\perp}^{\prime}(t)\right|}$, where $\rho \in\{ \pm 1\}$ is independent from $t$. We obtain

$$
\begin{aligned}
I(x) & :=\int_{T} \mathbb{1}_{K} f(x, \xi) d \omega_{\Omega}(z, \xi)=\frac{1}{2 \pi} \int_{T} \mathbb{1}_{K} f(x, \xi) \frac{\partial G}{\partial n}(\xi) d \sigma(\xi) \\
& =\frac{1}{2 \pi} \int_{0}^{1} \mathbb{1}_{K} f(x, \varphi(t))\left\langle\nabla G(\varphi(t)), \rho \frac{\varphi_{\perp}^{\prime}(t)}{\left|\varphi_{\perp}^{\prime}(t)\right|}\right\rangle\left|\varphi_{\perp}^{\prime}(t)\right| \mathrm{dt} \\
& =\frac{1}{2 \pi} \rho \int_{0}^{1} \mathbb{1}_{K} f(x, \varphi(t))\left\langle\nabla G(\varphi(t)), \varphi_{\perp}^{\prime}(t)\right\rangle \mathrm{dt}
\end{aligned}
$$

since $\left.\sigma\right|_{T}=\left|\varphi^{\prime}(t)\right| \mathrm{dt} \circ \varphi^{-1}=\left|\varphi_{\perp}^{\prime}(t)\right| \mathrm{dt} \circ \varphi^{-1}$ and $\frac{\partial G}{\partial n}(\varphi(t))=\left\langle\nabla G(\varphi(t)), \rho \frac{\varphi_{\perp}^{\prime}(t)}{\left|\varphi_{\perp}^{\prime}(t)\right|}\right\rangle$. We may assume that $\rho=1$. We set

$$
\begin{gathered}
g:[0,1] \rightarrow \mathbb{R}, g(t):=\left\langle\nabla G(\varphi(t)), \varphi_{\perp}^{\prime}(t)\right\rangle, \\
V:=\left\{(x, t) \in \mathbb{R}^{n} \times \mathbb{R} \mid-1-\varepsilon<t<1+\varepsilon \text { and }(x, \varphi(t)) \in U\right\}, \\
L:=\left\{(x, t) \in \mathbb{R}^{n} \times \mathbb{R} \mid 0 \leq t \leq 1 \text { and }(x, \varphi(t)) \in K\right\}, \\
h: V \rightarrow \mathbb{R}, h(x, t):=f(x, \varphi(t)) .
\end{gathered}
$$

Then $V$ is open, $L$ is globally subanalytic and relatively compact in $V$, and $h$ is real analytic on $V$. We get

$$
I(x)=\frac{1}{2 \pi} \int_{0}^{1} \mathbb{1}_{L} h(x, t) g(t) \mathrm{dt} .
$$

By a similar argument as in the proof of Example $1.4 \mathrm{~b}$ ) and by changing the limits of integration if necessary, we may replace $V$ by an open box $Q$ such that $h$ can be expanded into a power series that converges on a neighbourhood of $\bar{Q}$. Moreover, we may assume that either $0 \notin \overline{\pi(Q)}$ (where $\pi: \mathbb{R}^{n} \times \mathbb{R} \rightarrow \mathbb{R}$ is the projection onto the last coordinate) or there is some $\eta \in \mathbb{R}^{n}$ such that $(\eta, 0)$ is the center of $Q$. 
Case 1: $0 \notin \overline{\pi(Q)}$. It suffices to consider the case $\pi(Q)=] a, c[$ where $0<a \leq 1$. Setting $b:=\min \{1, c\}$ we have

$$
I(x)=\frac{1}{2 \pi} \int_{a}^{b} \mathbb{1}_{L} h(x, t) g(t) \mathrm{dt} .
$$

Since $G$ has a real analytic extension to a neighbourhood of $\varphi([a, b])$ we see that $g$ has a real analytic extension to a neighbourhood of $[a, b]$ and we get by Example 1.4 b) that $I$ is definable in the o-minimal structure $\mathbb{R}_{\text {an }}$ which is a reduct of $\mathbb{R}_{\mathcal{Q}}$.

Case 2: $0 \in \overline{\pi(Q)}$. Then $\pi(Q)=]-c, c[$ for some $c>0$. Setting $b:=\min \{1, c\}$ we obtain that

$$
I(x)=\frac{1}{2 \pi} \int_{0}^{b} \mathbb{1}_{L} h(x, t) g(t) \mathrm{dt} .
$$

If $\varphi(0) \notin \operatorname{Sing}(\partial \Omega)$, then $g$ has a real analytic extension to a neighbourhood of $[0, b]$ and we get again by Example $1.4 \mathrm{~b}$ ) that $I$ is definable in $\mathbb{R}_{\mathrm{an}}$. So we assume that $\varphi(0) \in \operatorname{Sing}(\partial \Omega)$. Applying [21, Theorem 3.2] to the Poisson kernel $K_{z}$, we find a quadratic domain $\widehat{U} \subset \mathbf{L}$ with $\bar{T} \backslash\{0\} \subset \widehat{U}$ (note that $T \subset \mathbb{C} \backslash \mathbb{R}_{\leq 0}$ ) such that $G=K_{z}-H_{K_{z}}$ can be extended to the real part of a function $k \in \mathcal{Q}(\widehat{U})$. Here $\mathbf{L}$ denotes the Riemann surface of the logarithm (see [21, Section 1]). Identifying $\mathbb{R}^{2}$ with $\mathbb{C}$ we have $\varphi(t)=\psi(t)+i \chi(t) \in \mathcal{O}(B(0,1+\varepsilon))$ (where $(\psi, \chi)$ is from (i) in the description of $T)$. By the Cauchy-Riemann equations we get

$$
k^{\prime}(\varphi(t))=\frac{\partial G}{\partial x}(\varphi(t))-i \frac{\partial G}{\partial y}(\varphi(t))
$$

and therefore

$$
g(t)=\operatorname{Re}\left(k^{\prime}(\varphi(t)) i \varphi^{\prime}(t)\right)=\operatorname{Re}\left((i k \circ \varphi)^{\prime}(t)\right) .
$$

So

$$
I(x)=\frac{1}{2 \pi} \int_{0}^{b} 1_{L} h(x, t) \operatorname{Re}\left((i k \circ \varphi)^{\prime}(t)\right) \mathrm{dt} .
$$

By (the arguments of) [22, Definition 5.16 and Corollary 5.17] we see that $i k \circ \varphi \in \mathcal{Q}$. So we finally get that

$$
I(x)=\frac{1}{2 \pi} \int_{0}^{b} \mathbb{1}_{L} h(x, t) \operatorname{Re}\left(H^{\prime}(t)\right) \mathrm{dt}
$$


where $H \in \mathcal{Q}\left(U^{\prime}\right)$ and $U^{\prime}$ is a standard quadratic domain with $] 0, b+\delta\left[\subset U^{\prime}\right.$ for some $\delta>0$. There is $H_{1}(t) \in \mathcal{Q}\left(U^{\prime}\right)$ and there is a finite sum $H_{2}(t)=\sum_{i=1}^{M} b_{i} t^{-\beta_{i}}$, where $\beta_{i}>0$ and $b_{i} \in \mathbb{C}^{*}$, such that $H^{\prime}(t)=H_{1}(t)+H_{2}(t)$. Note that $-\beta_{i}>-1$ for all $i$. Let $(\eta, 0)$ be the center of $Q$. Let $h(x, t)=\sum_{n=0}^{\infty} a_{n}(x) t^{n}$ be the power series expansion of $h$ on $Q$. Let $T H_{1}=\sum_{k=0}^{\infty} a_{k} t^{\alpha_{k}}\left(\right.$ where $\left\{\alpha_{k}: k \geq 0\right\}=\operatorname{supp}\left(T H_{1}\right)$ and $\left.\alpha_{k} \nearrow \infty\right)$ be the asymptotic expansion of $H_{1}$ (see [22, Definition 2.6]). For $n \in \mathbb{N}_{0}$ we define

$$
H_{1, n}: U^{\prime} \rightarrow \mathbb{C}, z \mapsto \int_{\gamma_{z}} H_{1}(\zeta) \zeta^{n} d \zeta,
$$

where $\gamma_{z}:[0,1] \rightarrow \mathbf{L}, s \mapsto(s|z|, \arg z)$. We see (compare with [38, (5) on p. 48]) that $H_{1, n} \in \mathcal{Q}\left(U^{\prime}\right)$ and that $T H_{1, n}=\sum_{k=0}^{\infty} \frac{a_{k}}{\alpha_{k}+n+1} t^{\alpha_{k}+n+1}$. Moreover, after shrinking $U^{\prime}$ if necessary, we may assume that there is some $C>0$ such that $\left|H_{1}(z)\right| \leq C$ for all $z \in U^{\prime}$ and that $U^{\prime}$ is bounded; i.e. there is some $D>0$ such that $U^{\prime} \subset B_{\mathbf{L}}(D)$. Then $\left|H_{1, n}(z)\right| \leq D C|z|^{n}$ for all $z \in U^{\prime}$. We set $\widehat{I}_{n}:=H_{1, n} z^{-n}$. Then $\widehat{I}_{n} \in \mathcal{Q}\left(U^{\prime}\right)$ with $T \widehat{I I}_{n}:=\sum_{k=0}^{\infty} \frac{a_{k}}{\alpha_{k}+n+1} z^{\alpha_{k}+1}$ and $\left|\widehat{I}_{n}\right| \leq D C$ for all $z \in U^{\prime}$. Repeating the same argument for $t^{-\alpha_{N}}\left(H_{1}-\sum_{k=0}^{N-1} a_{k} t^{\alpha_{k}}\right)$ and all $N \in \mathbb{N}$ we see with [22, Definition 5.1 and Proposition 2.15] that

$$
\widehat{I}: \widehat{Q} \times U^{\prime} \rightarrow \mathbb{C}, \widehat{I}(x, t, z):=\sum_{n=0}^{\infty} a_{n}(x) t^{n} \widehat{I}_{n}(z),
$$

is an element of $\mathcal{Q}\left(\widehat{Q} \times U^{\prime}\right)$ where $\widehat{Q}$ is a polydisc in $\mathbb{C}^{n+1}$ which has the same center as $Q$ and fulfils $\widehat{Q} \cap \mathbb{R}^{n+1} \supset \bar{Q}$.

Let

$$
\widetilde{I}(x, t, z):=\sum_{i=0}^{M} b_{i}\left(\sum_{n=0}^{\infty} \frac{a_{n}(x)}{n-\beta_{i}+1} t^{n}\right) z^{-\beta_{i}+1} .
$$

Then $\widetilde{I} \in \mathcal{Q}\left(\widehat{Q} \times U^{\prime}\right)($ see [22, Remark 5.2]). By construction, the antiderivative of $h(x, t) H^{\prime}(t)$ with respect to $t$ is given by $\widehat{I}(x, t, t)+\widetilde{I}(x, t, t)$ and we get by a similar argument as in the proof of Example $1.4 \mathrm{~b}$ ), part (iii), that $I(x)$ is definable in $\mathbb{R}_{\mathcal{Q}}$. 
To establish subanalytical tameness, we need convergence of the green function. This is the case if the domain in question is a polygon (see [18, Section 4]). Then the green function restricted to the boundary is definable in the o-minimal structure $\mathbb{R}_{\mathrm{an}}^{\mathbb{R}}$ which is the expansion of $\mathbb{R}_{\mathrm{an}}$ by the power functions $x \mapsto x^{\lambda}$ for arbitrary real $\lambda$ (see [9] and [27] for this o-minimal structure). Note that functions definable in $\mathbb{R}_{\mathrm{an}}^{\mathbb{R}}$ are exactly the functions with are piecewise given by convergent generalized power series as considered in [10] with support (i.e., the set of exponents, see [10, p. 4377]) contained in a finitely generated monoid over the nonnegative numbers. We also need "bad" approximation by rational numbers if the angle at a singular boundary point of the polygon is an irrational multiple of $\pi$ in order to keep convergence when integrating globally subanalytic functions with respect to the harmonic measure. These irrational numbers with "bad" approximation are called Diophantine. They play also a crucial role in dynamical systems (see Siegel [34], Ilyashenko [16], Carleson and Gamelin [3, II. 6] and Milnor [28, $\$ 11$ and Appendix C]). We give the definition below. In Theorem B we show that the harmonic measure of a polygon where the irrationals described above are Diophantine is subanalytically tame. To establish this result we use the preparation theorem for globally subanalytic functions (see [25, Théorème 1] and [26, Section 3]).

Definition 3.1 (See for example [28, p. 119].) An irrational number $\alpha$ is called Diophantine if there are positive constants $\varepsilon$ and $\sigma$ such that $\left|\alpha-\frac{p}{q}\right|>\varepsilon|q|^{-\sigma}$ for all $\frac{p}{q} \in \mathbb{Q}$. Note that necessarily $\sigma \geq 2$ by the theory of continued fractions. Irrational numbers that are not Diophantine are called Liouville numbers.

Remark 3.2 a) By Liouville's Approximation Theorem (see for example [28, p. 119]), every irrational real algebraic number is Diophantine. By Roth's Theorem (see for example [28, p. 120]), any $\sigma>2$ can be chosen.

b) The set of Liouville numbers has Lebesgue measure 0 (see for example [28, p. 222]).

We need the following lemma in the proof of Theorem B below.

Lemma 3.3 Let $\alpha \in \mathbb{R} \backslash \mathbb{Q}$ be Diophantine. Let $p \in \mathbb{N}$. Then there are positive constants $\mu, \rho$ such that $\left|k \alpha-\frac{m}{p}\right| \geq \mu k^{-\rho}$ for all $k \in \mathbb{N}$ and $m \in \mathbb{Z}$.

Proof Let $\varepsilon, \sigma$ be as in Definition 3.1. Then $\left|k \alpha-\frac{m}{p}\right|=k\left|\alpha-\frac{m}{k p}\right| \geq \varepsilon p^{-\sigma} k^{-\sigma+1}$.

Definition 3.4 Let $P$ be a polygon. We say that $P$ is Diophantine if for every $x \in \operatorname{Sing}(\partial P)$ either $\varangle_{x} P / \pi \in \mathbb{Q}$ or $\varangle_{x} P / \pi$ is Diophantine. 
In Theorem B below we will show that the harmonic measure for a Diophantine polygon is subanalytically tame. By the explanation above, we have to integrate certain functions with parameters that are definable in $\mathbb{R}_{\mathrm{an}}^{\mathbb{R}}$. As mentioned in the introduction, Soufflet $[35,36]$ has partial results on this problem as follows. By [27] a function definable in $\mathbb{R}_{\mathrm{an}}^{\mathbb{R}}$ is piecewise given by a finite composition of globally subanalytic functions and power functions. Soufflet has shown that the integral with parameters of such a composition is definable in $\mathbb{R}_{\mathrm{an} \text {,exp }}$ if the exponents of the involved power functions are outside a certain small set of Lebesgue measure 0 . To establish this result he uses, similarly to the approach of Comte, Lion and Rolin, the preparation theorem for functions definable in $\mathbb{R}_{\mathrm{an}}^{\mathbb{R}}$ (see [25, Théorème 3]). Since the exponents may change (in a polynomial way) by preparing a given function (see [35, Lemma 3.1]), and since the Diophantine numbers are not closed under addition and multiplication, we have to explicitely compute the preparations in the proof of Theorem B below. And then a little extra work is necessary compared to [35, Proposition 2.4] (for example, the prepared function $1+t+t^{\alpha}$ where $\alpha$ is Diophantine is not covered by [35, Definition 2.1]).

Theorem B Let $P$ be a Diophantine polygon. Let $z \in P$. Then the harmonic measure $w_{\Omega}(z,-)$ is subanalytically tame with subanalytically integrating o-minimal structure $\mathbb{R}_{\text {an,exp. }}$.

Proof As in the proof of Proposition 2.3 we assume that $P$ is simply connected. Let $F: P \rightarrow B(0,1)$ be a biholomorphic map with $F(z)=0$. By [18, Proposition 2 in Section 4], we have that $F$ is definable in $\mathbb{R}_{\mathrm{an}}^{\mathbb{R}}$. Then the Green function $G_{z}$ is given by $G_{z}=-\log |F|$ (see [14, Theorem II. 2.1]) and therefore also definable in $\mathbb{R}_{\mathrm{an}}^{\mathbb{R}}$. Using the subanalytic preparation theorem (see [25, Théorème 1] and [26, Section 3]), and by doing the same reduction steps as at the beginning of Theorem A (with a stratum $T \subset \partial \Omega$ and $0 \in \bar{T} \backslash T$ as the relevant and possibly singular boundary point), we have to show that

$$
I: B \rightarrow \mathbb{R}, x \mapsto \int_{\varphi(x)}^{\psi(x)} f(x, t) H^{\prime}(t) \mathrm{dt},
$$

is definable in $\mathbb{R}_{\mathrm{an}, \exp }$, where

(1) $B \subset \mathbb{R}^{n}$ is a globally subanalytic set, called the basis,

(2) $\varphi, \psi: B \rightarrow[0,1]$ are globally subanalytic functions with $\varphi<\psi$, 
(3) $f: C:=\{(x, t) \in B \times \mathbb{R} \mid \varphi(x)<t<\psi(x)\} \rightarrow \mathbb{R}$ is globally subanalytic of the following form (see [25, Théorème 1] and [26, Section 3]):

There is $r \in \mathbb{Q}$ such that $f=A(t-\Theta(x))^{r} U$ where the functions $A, \Theta$ and $U$ fulfil these conditions:

(a) $A$ is a globally subanalytic function on the basis $B$.

(b) $\Theta$ is a globally subanalytic function on $B$. Moreover, either $\Theta \equiv 0$ or $\left|\frac{t}{\Theta}\right|$ takes values in a compact subset of $] 0, \infty[$. We may assume that $\Theta>0$ or $\Theta<0$ if $\Theta \neq 0$.

(c) $U(x, t)=u\left(\phi_{1}, \ldots, \phi_{s}, \alpha, \beta\right)$ where $u(w, y)$ is a real power series convergent on $(B(0,2))^{s+2}\left(w=\left(w_{1}, \ldots, w_{s}\right), y=\left(y_{1}, y_{2}\right)\right)$ taking values in a compact subset of $] 0, \infty\left[\right.$, where $\alpha:=\left(\frac{t-\Theta}{a}\right)^{\frac{1}{p}}$ and $\beta:=\left(\frac{b}{t-\Theta}\right)^{\frac{1}{p}}$ are functions with values in $[0,1]$ (with $p \in \mathbb{N}$ and $a, b$ globally subanalytic functions on $B$ ), and where $\phi_{1}, \ldots, \phi_{s}$ are globally subanalytic functions on the basis $B$ with values in $[0,1]$.

and

(4) $H$ is a generalized power series (in the sense of [10]) convergent on $[0,1+\epsilon]$ for some $\epsilon>0$ and definable in $\mathbb{R}_{\mathrm{an}}^{\mathbb{R}}$. Moreover, the following holds by (the proof of) [18, Proposition 2 in Section 4] and by the fact that $G_{z}=-\log |F|$ :

Either

(i) $0 \notin \operatorname{Sing}(\partial \Omega)$ or $\partial_{0} P / \pi \in \mathbb{Q}$ and then $H$ is a Puiseux series. In particular $H$ is definable in $\mathbb{R}_{\mathrm{an}}$,

or

(ii) $\quad \alpha:=\partial_{0} P / \pi \in \mathbb{R} \backslash \mathbb{Q}$ and then $\operatorname{supp}(H) \subset \mathbb{N}_{0}+\mathbb{N} \alpha$.

Note that we can assume by Proposition 2.3 that $I$ is well-defined (i.e. finite). If (i) holds we see with [4, Théorème 1'] and [26, Théorème 1] that $I(x)$ is definable in $\mathbb{R}_{\mathrm{an} \text {,exp }}$. So we assume that (ii) holds. Note that $\alpha:=\partial_{0} P / \pi$ is Diophantine by assumption.

We can write $H^{\prime}(t)=H_{1}(t)+H_{2}(t)$ such that $H_{1}(t)$ is a generalized power series convergent on $[0,1+\varepsilon]$ with $\operatorname{supp}\left(H_{1}(t)\right) \subset \mathbb{N}_{0}+\mathbb{N} \alpha$ and $H_{2}(t)=\sum_{i=1}^{N} b_{i} t^{\beta_{i}}$ where $\beta_{i}<0$ and $\beta_{i} \in \mathbb{N} \alpha-1$. 
We show that

$$
B \rightarrow \mathbb{R}, x \mapsto \int_{\varphi(x)}^{\psi(x)} f(x, t) H_{1}(t) \mathrm{dt},
$$

is definable in $\mathbb{R}_{\mathrm{an}, \exp }$. Therefore it is sufficient to show that there is a function on $C$ which is definable in $\mathbb{R}_{\mathrm{an}, \exp }$ and which is the antiderivative of $f(x, t) H^{\prime}(t)$ with respect to $t$. By the linearity of the integral, we may assume by condition (a) in (3) that the function $A$ equals 1. Moreover, it is enough to show the claim for $\tilde{f}(x, w, t):=(t-\Theta(x))^{r} u(w, \alpha, \beta)$ instead of $f(x, t)$ since we can plug in $\phi_{1}, \ldots, \phi_{s}$ after integrating. Let

$$
u(w, y)=\sum_{n \in \mathbb{N}_{0}^{2}} A_{n}(w) y^{n}
$$

be the power series expansion of $u$. Moreover, let

$$
H_{1}(t)=\sum_{(\ell, k) \in \mathbb{N}_{0} \times \mathbb{N}} a_{(\ell, k)} t^{\ell+k \alpha}
$$

be the expansion of $H_{1}$. We prepare the functions $f$ and $H_{1}$ simultaneously. We have to follow the change of the exponents carefully.

Case 1: $\Theta \not \equiv 0$.

Applying a cell decomposition (see [6, Chapter 3]) to $C$ we may assume that either $\left|\frac{t}{\Theta(x)}\right| \leq \frac{2}{3}$ or $\frac{2}{3}<\left|\frac{t}{\Theta(x)}\right|<\frac{3}{2}$ or $\left|\frac{t}{\Theta(x)}\right| \geq \frac{3}{2}$ for all $(x, t) \in C$.

Subcase 1.1: $\frac{2}{3}<\left|\frac{t}{\Theta(x)}\right|<\frac{3}{2}$ for all $(x, t) \in C$.

Subcase 1.1.1: $\Theta<0$.

We set $\tau:=t-\frac{\Theta(x)}{3}>0$. We will integrate with respect to $\tau$. We have

$$
\tau=t-\frac{\Theta(x)}{3}=t\left(1-\frac{\Theta(x)}{3 t}\right), t=\tau\left(1+\frac{\Theta(x)}{3 \tau}\right), t-\Theta(x)=\tau\left(1-\frac{2}{3} \frac{\Theta(x)}{\tau}\right) .
$$

By the imposed conditions we obtain that

$$
|\tau| \leq \frac{3}{2},\left|\frac{\Theta(x)}{\tau}\right| \leq 1,\left|\frac{\tau}{a(x)}\right| \leq 1,\left|\frac{b(x)}{\tau}\right| \leq \frac{5}{3} .
$$


So all the relevant data are bounded. By binomial expansion, and by applying [10, Lemma 6.5] and a compactness argument, we can partition $C$ into finitely many globally subanalytic cells $C_{1}, \ldots, C_{k}$ such that for each $1 \leq i \leq k$ the following holds.

There are positive constants $\kappa_{i}, \lambda_{i}, \mu_{i}$ and a real power series $u_{i}(w, y)\left(y=\left(y_{1}, y_{2}, y_{3}\right)\right)$ convergent on $(B(0,2))^{n} \times B\left(0, \kappa_{i}\right) \times B\left(0, \lambda_{i}\right) \times B\left(0, \mu_{i}\right)$ such that

$$
\tilde{f}(x, w, t)=(t-\Theta(x))^{r} u(w, \alpha, \beta)=\tau^{r} u_{i}\left(w,\left(\frac{\tau}{a(x)}\right)^{\frac{1}{p}},\left(\frac{b(x)}{\tau}\right)^{\frac{1}{p}}, \frac{\Theta(x)}{\tau}\right)
$$

and $\left|\frac{\tau}{a(x)}\right|^{\frac{1}{p}} \leq \varepsilon_{i} \kappa_{i},\left|\frac{b(x)}{\tau}\right|^{\frac{1}{p}} \leq \varepsilon_{i} \lambda_{i},\left|\frac{\Theta(x)}{\tau}\right| \leq \varepsilon_{i} \mu_{i}$ for all $(x, t) \in C_{i}$ and some $0<\varepsilon_{i}<1$.

And, there are positive constants $\rho_{i}, \sigma_{i}$ and $h_{i}\left(z_{1}, z_{2}\right) \in \mathbb{R}\left\{z_{1}, z_{2}^{*}\right\}_{\rho_{i}, \sigma_{i}}$ (see the notation of [10, pp. 4391-4395]) such that

$$
H_{1}(t)=h_{i}\left(\frac{\Theta(x)}{\tau}, \tau\right)
$$

and $\left|\frac{\Theta(x)}{\tau}\right| \leq \delta_{i} \rho_{i},|\tau| \leq \delta_{i} \sigma_{i}$ for all $(x, t) \in C_{i}$ and some $0<\delta_{i}<1$. Note that $\operatorname{supp}\left(h_{i}\right) \subset \mathbb{N}_{0} \times\left(\mathbb{N}_{0}+\mathbb{N} \alpha\right)$. So $h_{i}$ is definable in $\mathbb{R}_{\text {an }}^{\mathbb{R}}$. Let

$$
u_{i}(w, y)=\sum_{n \in \mathbb{N}_{0}^{3}} B_{n}(w) y^{n} \text { and } h_{i}\left(z_{1}, z_{2}\right)=\sum_{(m, \ell, k) \in \mathbb{N}_{0}^{2} \times \mathbb{N}} c_{(m, \ell, k)} z_{1}^{m} z_{2}^{\ell+k \alpha}
$$

be the expansions of $u_{i}$ resp. $h_{i}$. We have

$$
u_{i}(w, y) h_{i}(z)=\sum_{\lambda \in \mathbb{N}_{0}^{5} \times \mathbb{N}} B_{n}(w) c_{(m, \ell, k)} y^{n} z_{1}^{m} z_{2}^{\ell+k \alpha}
$$

where $\lambda=(n, m, \ell, k) \in \mathbb{N}_{0}^{5} \times \mathbb{N}$ and $n=\left(n_{1}, n_{2}, n_{3}\right) \in \mathbb{N}_{0}^{3}$. For such $\lambda$ we set

$$
d_{\lambda}:=r+\frac{n_{1}}{p}-\frac{n_{2}}{p}-n_{3}-m+\ell+k \alpha+1
$$

and define

$$
V_{i}:=\sum_{\lambda \in \mathbb{N}_{0}^{5} \times \mathbb{N}} d_{\lambda}^{-1} B_{n}(w) c_{(m, \ell, k)} \bar{y}^{n} z_{1}^{m} z_{2}^{\ell+k \alpha} .
$$

Now, we use that $\alpha$ is Diophantine. By Lemma 3.3 we see that $V_{i} \in \mathbb{R}\left\{w, y, z_{1}, z_{2}^{*}\right\} \omega_{i}$ where

$$
\omega_{i}:=\left(1, \ldots, 1, \varepsilon_{i} \kappa_{i}, \varepsilon_{i} \lambda_{i}, \varepsilon_{i} \mu_{i}, \delta_{i} \rho_{i}, \delta_{i} \sigma_{i}\right) \in \mathbb{R}_{>0}^{s+5} .
$$

The support of $V_{i}$ is contained in a finitely generated monoid. Hence $V_{i}$ is definable in $\mathbb{R}_{\mathrm{an}}^{\mathbb{R}}$. Since

$$
\tau^{r+1} V_{i}\left(w,\left(\frac{\tau}{a(x)}\right)^{\frac{1}{p}},\left(\frac{b(x)}{\tau}\right)^{\frac{1}{p}}, \frac{\Theta(x)}{\tau}, \frac{\Theta(x)}{\tau}, \tau\right)
$$


is the antiderivative of $\tilde{f}(x, w, t) H_{1}(t)$ with respect to $t$ for $(x, t) \in C_{i}$ the theorem follows in Subcase 1.1.1.

Subcase 1.1.2: $\Theta>0$.

We set $\tau:=t-\Theta(x)$ and integrate with respect to $\tau$. We have

$$
t=\tau+\Theta(x)=\Theta(x)\left(1+\frac{\tau}{\Theta(x)}\right) .
$$

By the imposed conditions in Subcase 1.1 we have

$$
\Theta(x)<\frac{3}{2},\left|\frac{\tau}{\Theta(x)}\right|<\frac{1}{2} .
$$

By applying [10, Lemma 6.5] again, we can partition $C$ into finitely many globally subanalytic cells $C_{1}, \ldots, C_{k}$ such that for each $1 \leq i \leq k$ see following holds:

There are positive constants $\rho_{i}, \sigma_{i}$ and $h_{i}\left(z_{1}, z_{2}\right) \in \mathbb{R}\left\{z_{1}, z_{2}^{*}\right\}_{\rho_{i}, \sigma_{i}}$ such that

$$
H_{1}(z)=h_{i}\left(\frac{\tau}{\Theta(x)}, \Theta(x)\right)
$$

and $\left|\frac{\tau}{\Theta(x)}\right| \leq \delta_{i} \rho_{i},|\Theta(x)| \leq \delta_{i} \sigma_{i}$ for all $(x, t) \in C_{i}$ and some $0<\delta_{i}<1$. Note that $\operatorname{supp}\left(h_{i}\right) \subset \mathbb{N}_{0} \times\left(\mathbb{N}_{0}+\mathbb{N} \alpha\right)$. Hence $h_{i}$ is definable in $\mathbb{R}_{\mathrm{an}}^{\mathbb{R}}$. Let

$$
h_{i}\left(z_{1}, z_{2}\right)=\sum_{(m, \ell, k) \in \mathbb{N}_{0}^{2} \times \mathbb{N}} b_{(m, \ell, k)} z_{1}^{m} z_{2}^{\ell+k \alpha} .
$$

be the expansion of $h_{i}$. For $\lambda:=(n, m, \ell, k) \in \mathbb{N}_{0}^{4} \times \mathbb{N}$ we set $c_{\lambda}:=r+\frac{n_{1}-n_{2}+m p}{p}$. If $c_{\lambda} \neq-1$ we set $d_{\lambda}:=c_{\lambda}+1$. We define

$$
V_{i}:=\sum_{\left\{\lambda: c_{\lambda} \neq-1\right\}} d_{\lambda}^{-1} A_{n}(w) b_{(m, \ell, k)} y^{n} z_{1}^{m} z_{2}^{\ell+k \alpha}
$$

and

$$
D_{i}(w, x):=\sum_{\left\{\lambda: c_{\lambda}=-1\right\}} A_{n}(w) b_{(m, \ell, k)} a(x)^{-\frac{n_{1}}{p}} b(x)^{\frac{n_{2}}{p}} \Theta(x)^{-m+\ell+k \alpha} .
$$

Then $V_{i} \in \mathbb{R}\left\{w, y, z_{1}, z_{2}^{*}\right\}_{\omega_{i}}$ where $\omega_{i}:=\left(1, \ldots, 1, \delta_{i} \rho_{i}, \delta_{i} \sigma_{i}\right)$. Since the support of $V_{i}$ is contained in a finitely generated monoid we get that $V_{i}$ is definable in $\mathbb{R}_{\mathrm{an}}^{\mathbb{R}}$. The same holds for $D_{i}: B_{i} \rightarrow \mathbb{R}$ (where $B_{i}$ denotes the basis of $C_{i}$ ) by the same argument. Since

$$
\tau^{r+1} V_{i}\left(w,\left(\frac{\tau}{a(x)}\right)^{\frac{1}{p}},\left(\frac{b(x)}{\tau}\right)^{\frac{1}{p}}, \frac{\tau}{\Theta(x)}, \Theta(x)\right)+D_{i}(x, w) \sigma \log |\tau|
$$


is the antiderivative of $\tilde{f}(x, w, t) H_{1}(t)$ with respect to $t$ for $(x, t) \in C_{i}$ and suitable $\sigma \in\{ \pm 1\}$ the theorem follows in Subcase 1.1.2.

Subcase 1.2: $\left|\frac{t}{\Theta(x)}\right| \leq \frac{2}{3}$ for all $(x, t) \in C$.

We have

$$
\Theta(x)-t=\Theta(x)\left(1-\frac{t}{\Theta(x)}\right)
$$

We see that

$$
\left|\frac{\Theta(x)}{a(x)}\right| \leq 3,\left|\frac{b(x)}{\Theta(x)}\right| \leq \frac{5}{3}
$$

for all $(x, t) \in C$. Also $\Theta(x)$ is bounded by the imposed conditions in (b). Now we proceed as in Subcase 1.1.1 (integrating with respect to $t$ ). Note that coordinate transformation is only necessary for $\tilde{f}(x, w, t)$, not for $H_{1}(t)$.

Subcase 1.3: $\left|\frac{t}{\Theta(x)}\right| \geq \frac{3}{2}$ for all $(x, t) \in C$.

We write

$$
t-\Theta(x)=t\left(1-\frac{\Theta(x)}{t}\right)
$$

We see that

$$
\left|\frac{t}{a(x)}\right| \leq 3,\left|\frac{b(x)}{t}\right| \leq \frac{5}{3}
$$

for all $(x, t) \in C$. We proceed as in Subcase 1.1.1 or Subcase 1.2 (integrating with respect to $t$ ); again coordinate transformation is only necessary for $\tilde{f}(x, w, t)$.

Case 2: $\Theta \equiv 0$.

We integrate with respect to $t$, no coordinate transformations are necessary.

We can repeat the arguments replacing $H_{1}$ by $H_{2}$ and obtain the claim. 


\section{References}

[1] D.H. Armitage, S.J. Gardiner: Classical Potential Theory. Springer Monographs in Mathematics, Springer, 2001.

[2] E. Bierstone, P.D. Milman: Semianalytic and subanalytic sets. Inst. Hautes Études Sci. Publ. Math. 67 (1988), 5-42; doi:10.1007/BF02699126

[3] L. Carleson, T.W. Gamlin: Complex dynamics. Springer, 1993.

[4] G. Comte, J.-M. Lion, J.-P. Rolin: Nature log-analytique du volume des sousanalytiques. Illinois J. Math. 44, no.4 (2000), 884-888.

[5] L. van den Dries: A generalization of the Tarski-Seidenberg Theorem, and some non definability results. Bull. AMS 15 (1986), 189-193; doi:10.1090/S0273-0979-198615468-6

[6] L. van den Dries: Tame Topology and O-minimal Structures. London Math. Soc. Lecture Notes Series 248, Cambridge University Press, 1998.

[7] L. van den Dries, A. Macintyre, D. Marker: The elementary theory of restricted analytic functions with exponentiation. Annals of Math. (2) 140, no.1 (1994), 183-205; doi: $10.2307 / 2118545$

[8] L. van den Dries, C. Miller: On the real exponential field with restricted analytic functions. Israel J. Math. 85, no.1-3 (1994), 19-56; doi:10.1007/BF02758635

[9] L. van den Dries, C. Miller: Geometric categories and o-minimal structures. Duke Math. Journal 84, no. 2 (1996), 497-540; doi:10.1215/S0012-7094-96-08416-1

[10] L. van den Dries, P. Speissegger: The real field with convergent generalized power series. Trans. Amer. Math. Soc. 350, no.11 (1998), 4377-4421; doi:10.1090/S00029947-98-02105-9

[11] L. van den Dries, P. Speissegger: The field of reals with multisummable series and the exponential function. Proc. London Math. Soc. (3) 81, no.3 (2000), 513-565; doi:10.1112/S0024611500012648

[12] T.A. Driscoll, L.N. Trefethen: Schwarz-Christoffel Mapping. Cambridge University Press, 2002.

[13] D.B. Garnett: Applications of harmonic measure. University of Arkansas Lecture Notes in the mathematical Sciences. Vo. 8, Wiley, 1986.

[14] D.B. Garnett, R.E. Marshall: Harmonic Measure. Cambridge University Press, 2005.

[15] L.L. Helms: Introduction to potential theory. Wiley-Interscience, 1969.

[16] Y.S. Ilyashenko: The finiteness problem for limit cycles of polynomial vector fields on the plane, germs of saddle resonant vector fields and non-Hausdorff Riemann surfaces. Topology (Leningrad 1982), Lecture Notes in Math. 1060, 290-305, Springer 1984.

[17] Y.S. Ilyashenko: Finiteness Theorems for Limit Cycles. Translations of Mathemat. Monographs 94, American Mathematical Society, 1991. 
[18] T. Kaiser: Definability results for the poisson equation. Adv.Geom. 6 (2006), 627-644; doi:10.1515/ADVGEOM.2006.036

[19] T. Kaiser: Dirichlet regularity of subanalytic domains. Trans. Amer. Math. Soc. 360 (2008), 6573-6594; doi:10.1090/S0002-9947-08-04609-6

[20] T. Kaiser: The Riemann Mapping Theorem for semianalytic domains and o-minimality. Proc. Lond. Math. Soc. (3) 98, no. 2 (2009), 427-444; doi:10.1112/plms/pdn034

[21] T. Kaiser: The Dirichlet problem in the plane with semianalytic raw data, quasianalyticity and o-minimal structures. Duke Math. Journal 147, no. 2 (2009), 285-314; doi:10.1215/00127094-2009-012

[22] T. Kaiser, J.-P. Rolin, P. Speissegger: Transition maps at non-resonant hyperbolic singularities are o-minimal. Journal für die reine und angewandte Mathematik $\mathbf{6 3 6}$ (2009), 1-45; doi:10.1515/CRELLE.2009.081

[23] K. Kurdyka: On a subanalytic stratification satisfying a Whitney property with exponent 1. Real Algebraic Geometry, Proceedings Rennes, 1991. M. Coste ed., Lecture Notes in Math. 1524, 316-323, Springer, Berlin, Heidelberg, New York, 1992.

[24] R.S. Lehman: Development of the mapping function at an analytic corner. Pacific J. of Math. 7 (1957), 1437-1449.

[25] J.-M. Lion, J.-P. Rolin: Théorème de préparation pour les fonctions logarithmicoexponentielles Ann. Inst. Fourier 47, no.3 (1997), 859-884.

[26] J.-M. Lion, J.-P. Rolin: Intégration des fonctions sous-analytiques et volumes des sous-ensembles sous-analytiques. Ann. Inst. Fourier 48, no.3 (1998), 755-767.

[27] C. Miller: Expansions of the real field with power functions. Ann. Pure Appl. Logic 68 (1994), 79-94; doi:10.1016/0168-0072(94)90048-5

[28] J. Milnor: Dynamics in One Complex Variable. Vieweg, 1999.

[29] M. Parusinski: Subanalytic functions. Trans. Amer. Math. Soc. 344 (1994), 583-595; doi: $10.2307 / 2154496$

[30] M. Parusinski: On the preparation theorem for subanalytic functions. New developments in singularity theory (Cambridge, 2000), 193-215, NATO Sci.Se. II Math. Phys. Chem., 21, Kluwer Acad. Publ., Dordrecht, 2001.

[31] Ch. Pommerenke: Boundary Behaviour of Conformal Maps, Springer, 1992.

[32] J.-P. Rolin, P. Speissegger, A.J. Wilkie: Quasianalytic Denjoy-Carleman classes and o-minimality. J. Amer. Math. Soc. 16, no.4 (2003), 751-777; doi:10.1090/S0894-034703-00427-2

[33] M. Shiota: Geometry of subanalytic and semialgebraic sets. Birkhäuser, 1997.

[34] C.L. Siegel: Über die Normalform analytischer Differentialgleichungen in der Nähe einer Gleichgewichtslösung. Nach. Akad. Wiss. Göttingen, Math.-Phys. (1952), 21-30.

[35] R. Soufflet: Finiteness property for generalized Abelian integrals. Ann. Inst. Fourier 52, no.3 (2003), 767-785. 
[36] R. Soufflet: Propriétés oscillatoires des intégrals de $x^{\lambda}$-fonctions. C. R. Acad. Sci. Paris Ser. I Math. 333, no. 5 (2001), 461-464; doi:10.1016/S0764-4442(01)02094-8

[37] P. Speissegger: The Pfaffian closure of an o-minimal structure. J. Reine Angew. Math. 508 (1999), 189-211; doi:10.1515/crll.1999.508.189

[38] W. Wasow: Asymptotic development of the solution of Dirichlet's problem at analytic corners. Duke Math. Journal 24 (1957), 47-56; doi:10.1215/S0012-7094-57-02408-0

[39] A.J. Wilkie: Model completeness results for expansions of the ordered field of real numbers by restricted Pfaffian functions and the exponential function. Journal Amer. Math. Soc. 9, no.4 (1996), 1051-1094; doi:10.1090/S0894-0347-96-00216-0

Fakultät für Informatik und Mathematik, Universität Passau, 93040 Passau, Germany

tobias.kaiser@uni-passau.de

Received: 4 November 2009 Revised: 16 June 2010 